This is the peer reviewed version of the following article:

R. M. Morales, G. Li and W. Heath. Anti-windup and the preservation of robustness against structured norm-bounded uncertainty. International Journal of Robust and Nonlinear Control, vol. 24, no. 17, pp. 2640-2652, 2014.

, which has been published in final form at http://dx.doi.org/10.1002/rnc.3014. This article may be used for non-commercial purposes in accordance with Wiley Terms and Conditions for SelfArchiving." 


\title{
Anti-windup and the preservation of robustness against structured norm-bounded uncertainty
}

\author{
R. M. Morales ${ }^{1, * \dagger}$, G. $\mathrm{Li}^{2}$ and W. P. Heath ${ }^{3}$ \\ ${ }^{1}$ Control Systems Research Group, Department of Engineering, University of Leicester, Leicester, LE1 7RH, UK \\ ${ }^{2}$ School of Engineering, Computing and Mathematics, University of Exeter, Exeter, EX4 4QF, UK \\ ${ }^{3}$ Control Systems Centre, School of Electrical and Electronic Engineering, \\ University of Manchester, Manchester, M13 9PL, UK
}

\begin{abstract}
SUMMARY
Anti-windup systems are modified control structures, which are designed to compensate against the detrimental effects of saturations. This manuscript considers primarily the stability robustness of two well-known anti-windup structures. Sufficient conditions for the stability robustness of the anti-windup structures and optimal robustness against structured norm-bounded plant uncertainty are found. A saturated loop is said to be optimally robust if the constrained loop is as robust as its unconstrained counterpart. The robustness condition is shown to be less conservative than existing results on additive uncertainty. Although it is known that the conventional internal model control provides optimal robustness against additive unstructured uncertainty, this is not the case against the more general uncertainty structure. Copyright (C) 2013 John Wiley \& Sons, Ltd.
\end{abstract}

Received 1 July 2011; Revised 14 January 2013; Accepted 28 March 2013

KEY WORDS: robust control; anti-windup; nonlinear systems; IQCs

\section{INTRODUCTION}

A common nonlinearity encountered in most (if not all) practical control systems is actuator saturation, which can impose significant limitations on the robustness and the performance of a control system. To mitigate the deteriorations caused by saturation nonlinearities, extra loops on the control system can be added, which operate only when the system is saturated (and the desired control actions differ from the ones delivered to the actual plant). Such systems are known as 'anti-windup schemes' [3-5], and if properly designed, can offer significant compensation for the degradations in performance and robustness caused by saturations. A well-established anti-windup approach suggests designing the linear controller a priori and the anti-windup compensator a posteriori [6].

Arguably, the main purpose of anti-windup is to mitigate the degradations by saturations in terms of performance, and for the sake of simplicity in the design methods, many strategies have been developed under a nominal framework [7-10]. However, the robustness of anti-windup systems in the face of plant uncertainty also deserve significant attention as stability precedes any performance demands in any control application with more than one operating condition. Robustness of anti-windup systems has not been much explored in the literature with two notable exceptions:

\footnotetext{
*Correspondence to: R. M. Morales, Control Systems Research Group, Department of Engineering, University of Leicester, University Road, Leicester, LE1 7RH, UK.

†E-mail: rmm23@1e.ac.uk

¥ This paper is based on the works presented at the IFAC World Congress 2008 [1] and the IEEE Multi-Conference on Systems and Control 2011 [2].
} 
1. The Integral Quadratic Constraint (IQC) framework of [11] is naturally suited to the robustness analysis of anti-windup systems with norm-bounded uncertainty and a case study is discussed in Reference [12].

2. The authors in [13] consider the robustness of the Weston and Postlethwaite anti-windup scheme [14] to additive norm-bounded uncertainty. In particular, this paper derives a sufficient condition for robust stability and shows that the Internal Model Control structure (IMC) is optimally robust in the sense that it is guaranteed to preserve the robustness of the unsaturated loop, that is, when saturation limitations do not exist. An anti-windup system with such a desirable property will also be referred to as robustness-preserving anti-windup.

This work is inspired from the analytical results of [13]. We investigate the stability robustness of two well-known anti-windup schemes proposed by [14] and [15]. They will be referred to as Weston-Postlethwaite and IMC anti-windup schemes, respectively, and they are introduced briefly in Section 3 from a stability analysis point of view. The development of this work is constructed under the IQC framework of [11], whose notation and main result are provided in Section 2.

The first contribution of this work is the development of a sufficient robustness condition for the anti-windup structures considered in this manuscript. The condition is not only suitable to deal with the more general uncertainty structure but also proven to be less conservative than existing results in the literature for additive uncertainty. The main contribution is to find an expression for a robustness-preserving anti-windup scheme with more general uncertainty structure. The contributions can be found in Sections 4 and 5, respectively. The ideas are supported by means of examples and counterexamples in Section 6. The manuscript concludes with some final remarks in Section 7.

\section{INTEGRAL QUADRATIC CONSTRAINT NOTATION AND RESULTS}

One of the primary purposes of feedback is to deal with uncertainty. It has become widely accepted that the appropriate framework for representing uncertain dynamics for linear control systems is structured norm-bounded uncertainty [16-18]; that is, the input-output map from $u$ to $y$ of the plant to be controlled should be modelled as (Figure 1)

$$
\begin{aligned}
{\left[\begin{array}{c}
p_{\Delta} \\
y
\end{array}\right] } & =\left[\begin{array}{ll}
G_{11} & G_{12} \\
G_{21} & G_{22}
\end{array}\right]\left[\begin{array}{c}
q_{\Delta} \\
u
\end{array}\right] \\
q_{\Delta} & =\Delta p_{\Delta}
\end{aligned}
$$

where $G_{22}$ is the nominal plant model, $G_{12}, G_{21}$ and $G_{11}$ are known transfer function matrices that are used to describe the plant uncertainty in the frequency domain and the uncertainty is described by the element $\Delta$, which satisfies the following norm inequality

$$
\left\|\Delta p_{\Delta}\right\|_{\Gamma}^{2} \leqslant\left\|p_{\Delta}\right\|_{\Gamma}^{2} / \gamma_{\Delta}^{2}, \text { for all } p_{\Delta} \in L_{2}^{n}[0, \infty)
$$

Here, $\Gamma$ belongs to some specified class of positive definite symmetric matrix and $\gamma_{\Delta}$ is some positive scalar [11].

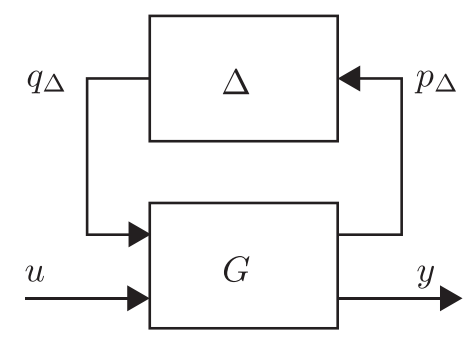

Figure 1. Plant model with structured norm-bounded uncertainty. 


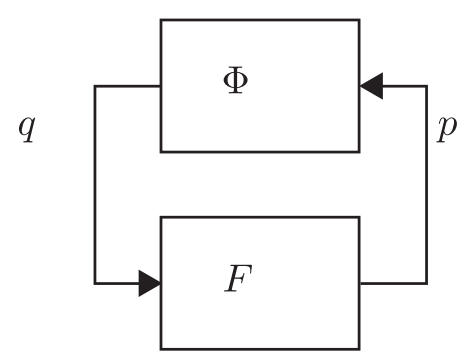

Figure 2. IQC set-up.

Under the IQC framework, it is said that an operator $\Phi \in \operatorname{IQC}(\Pi)$ for some self-adjoint operator $\Pi$ on $L_{2}^{2 n}[0, \infty)$ if for all $p, q \in L_{2}^{n}[0, \infty)$ with $q=\Phi(p)$, we have the relation

$$
\left\langle\left[\begin{array}{l}
p \\
q
\end{array}\right], \Pi\left[\begin{array}{l}
p \\
q
\end{array}\right]\right\rangle \geqslant 0
$$

with $\langle\cdot, \cdot\rangle$ denoting the $L_{2}$ inner product $[11,19]$.

Future developments of the ideas in this manuscript are constructed from the following stability lemma.

\section{Lemma 1}

Consider the closed-loop system depicted in Figure 2 described by

$$
\begin{aligned}
& q=\Phi(p) \\
& p=F q
\end{aligned}
$$

$F$ is some LTI stable transfer matrix. $\Phi$ is a norm-bounded operator that encapsulates any nonlinearity or uncertainty in the loop satisfying (3). Assume that the upper left and the lower right corner of $\Pi(j \omega)$ are positive semi-definite and negative semi-definite, respectively. In addition, assume the loop in question is well-posed. Stability is guaranteed in the input-output sense that [20] provided

$$
\left[\begin{array}{c}
F(j \omega) \\
I
\end{array}\right]^{*} \Pi(j \omega)\left[\begin{array}{c}
F(j \omega) \\
I
\end{array}\right]<0, \text { for all } \omega
$$

Proof

See [11].

Throughout this manuscript, we assume that both the nominal plant and the actual plant are open loop stable. We also assume that the closed-loop system without saturation is both nominally and robustly stable. For more information on the material presented in this section, refer to [11] and [19].

\section{ANTI-WINDUP SCHEMES}

\subsection{The Weston-Postlethwaite scheme}

The work [14] proposes the anti-windup scheme depicted in Figure 3. The design of the anti-windup element is carried out through the element $M$. The signal $r$ denotes the set point and $d$ denotes a disturbance signal. The linear controller is structured as $K=\left[K_{1} K_{2}\right]$. The operator sat(·) denotes term-by-term saturation so that the $i$ th element of $u=\operatorname{sat}(x)$ is given by

$$
u_{i}(t)= \begin{cases}-1 & \text { for } x_{i}(t) \leqslant-1 \\ x_{i}(t) & \text { for }-1<x_{i}(t)<1 \\ 1 & \text { for } 1 \leqslant x_{i}(t)\end{cases}
$$




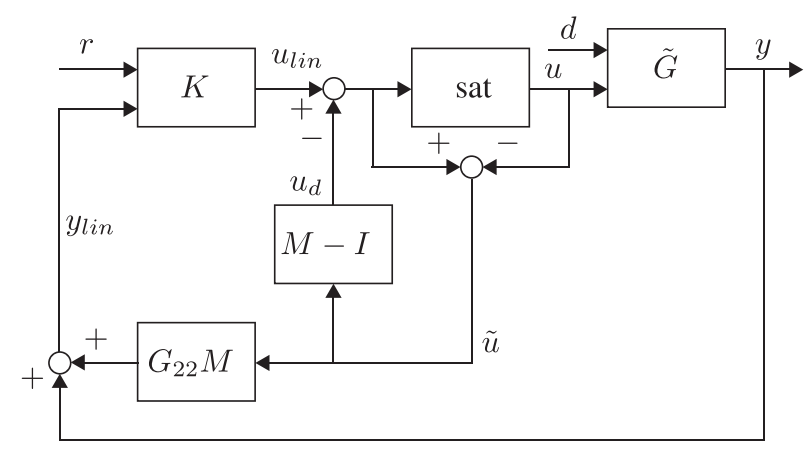

Figure 3. The anti-windup scheme of [14].

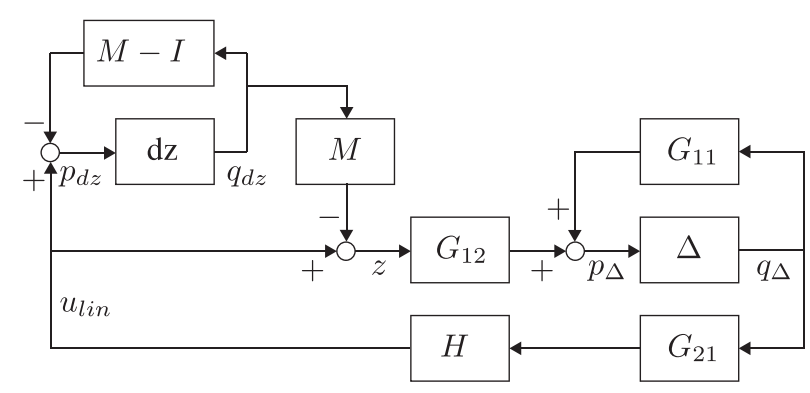

Figure 4. Equivalent scheme for stability analysis.

For the purposes of stability analysis, it is not necessary to consider either the reference signal $r$ or the exogenous disturbance signal $d$.

In this case, simple block diagram manipulation allows us to reduce the system to that depicted in Figure 4, where

$$
H=\left(I-K_{2} G_{22}\right)^{-1} K_{2}
$$

and the scheme is governed by the following relations

$$
\begin{aligned}
u_{l i n} & =H G_{21} q_{\Delta} \\
q_{\Delta} & =\Delta p_{\Delta} \\
p_{\Delta} & =G_{11} q_{\Delta}+G_{12} z \\
z & =u_{l i n}-M q_{d z} \\
q_{d z} & =\mathrm{dz}\left(p_{d z}\right) \\
p_{d z} & =u_{l i n}-(M-I) q_{d z}
\end{aligned}
$$

Here, $\operatorname{dz}(\cdot)$ denotes the dead zone operator defined as

$$
\mathrm{dz}(x)=x-\operatorname{sat}(x)
$$

Note that we have substituted the notation $q_{d z}$ and $z$ in (8) for $\tilde{u}$ and $u$, respectively. Also, note from the equivalent diagram that we must have $M(\infty)=I$ so that the feedback around the dead zone nonlinearity is strictly proper. For this work, we will assume that $M(\infty)=I$ is a requirement.

\subsection{Internal model control anti-windup}

The anti-windup scheme of [15] is depicted in Figure 5 for the purpose of stability analysis hence ignoring the set point and any feedforward signals. The anti-windup strategy is determined by the 


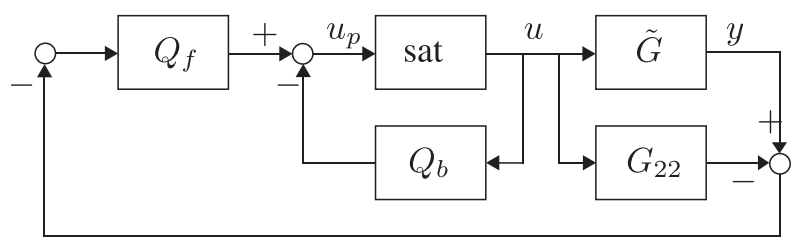

Figure 5. The IMC anti-windup scheme of [15].

choice of the elements $Q_{f}$ and $Q_{b}$. The scheme is based on the conventional IMC structure where the control input is given by

$$
u=-Q y+Q G_{22} u
$$

The linear controller $Q$ is designed via standard IMC principles [21] to achieve a desired performance and robustness level under a linear regime. The choice of the anti-windup elements must then obey the following relation

$$
Q=\left(I+Q_{b}\right)^{-1} Q_{f}
$$

The IMC anti-windup structure is governed by the following equations:

$$
\begin{aligned}
u & =\operatorname{sat}\left(u_{p}\right) \\
u_{p} & =-Q_{f} y+Q_{f} G_{22} u-Q_{b} u
\end{aligned}
$$

It is well-understood [22] that many anti-windup schemes are formally equivalent (of course, different structures are appropriate for different design procedures). The Weston-Postlethwaite and the IMC anti-windup schemes are equivalent if $M$ is invertible, and we set

$$
\begin{aligned}
Q & =-\left(I-K_{2} G_{22}\right)^{-1} K_{2} \\
Q_{f} & =M^{-1} Q \\
Q_{b} & =M^{-1}-I
\end{aligned}
$$

A special case occurs when $Q_{b}=0$. This corresponds to the choice $M=I$, and [13] refers only to this specific case.

\section{ROBUSTNESS ANALYSIS}

This section provides a robustness guarantee (Theorem 1) for the anti-windup scheme of Weston and Postlethwaite against structured plant uncertainty expressed in the more general Linear Fractional Transformation form. In Section 4.2, we discuss the special case when the plant uncertainty is additive and unstructured.

The norm-bounded uncertainty operator $\Delta$ is described by means of $\Gamma$ under the following IQC

$$
\left\langle\left[\begin{array}{l}
p_{\Delta} \\
q_{\Delta}
\end{array}\right],\left[\begin{array}{cc}
\Gamma & 0 \\
0 & -\gamma_{\Delta}^{2} \Gamma
\end{array}\right]\left[\begin{array}{l}
p_{\Delta} \\
q_{\Delta}
\end{array}\right]\right\rangle \geqslant 0
$$

Also, we will admit multipliers in the sector bound for the dead zone exploiting the following IQC

$$
\left\langle\left[\begin{array}{l}
p_{d z} \\
q_{d z}
\end{array}\right],\left[\begin{array}{cc}
0 & W^{*} \\
W & -W-W^{*}
\end{array}\right]\left[\begin{array}{l}
p_{d z} \\
q_{d z}
\end{array}\right]\right\rangle \geqslant 0
$$

for all $p_{d z}, q_{d z}$ such that $q_{d z}=\mathrm{dz}\left(p_{d z}\right)$. The class of admissible $W$ was considered for the SISO case by [23], and for the multivariable case by [24] and [25]. By straight application of Lemma 1, the following robustness criterion for the Weston-Postlethwaite structure is obtained. 
Theorem 1

The Weston-Postlethwaite structure with plant uncertainty (1) is guaranteed stable provided $L_{S}(j \omega)<0$ for all $\omega$ where

$$
L_{S}=\left[\begin{array}{ll}
L_{11} & L_{21}^{*} \\
L_{21} & L_{22}
\end{array}\right]
$$

and

$$
\begin{aligned}
& L_{11}=M^{*} G_{12}^{*} \Gamma G_{12} M-M^{*} W^{*}-W M \\
& L_{21}=G_{21}^{*} H^{*} W^{*}-\Theta^{*} \Gamma G_{12} M \\
& L_{22}=\Theta^{*} \Gamma \Theta-\gamma_{\Delta}^{2} \Gamma
\end{aligned}
$$

and

$$
\Theta=G_{11}+G_{12} H G_{21}
$$

Proof

Expressing the anti-windup structure in the feedback loop considered in Lemma 1 takes the form (4) with

$$
\begin{aligned}
& p=\left[\begin{array}{c}
p_{d z} \\
p_{\Delta}
\end{array}\right], q=\left[\begin{array}{c}
q_{d z} \\
q_{\Delta}
\end{array}\right] \\
& \Phi=\left[\begin{array}{ll}
\mathrm{dz} & \\
& \Delta
\end{array}\right], F=\left[\begin{array}{cc}
I-M & H G_{21} \\
-G_{12} M & \Theta
\end{array}\right]
\end{aligned}
$$

We have $\Phi \in \operatorname{IQC}(\Pi)$ with

$$
\Pi=\left[\begin{array}{cccc}
0 & 0 & W^{*} & 0 \\
0 & \Gamma & 0 & 0 \\
W & 0 & -W-W^{*} & 0 \\
0 & 0 & 0 & -\gamma_{\Delta}^{2} \Gamma
\end{array}\right]
$$

Condition (5) reduces to $L_{S}(j \omega)<0$ for all $\omega$.

Notice that Theorem 1 is easily extended to the IMC anti-windup structure by means of (13). Also, by considering static multipliers for both the uncertainty and the nonlinearity, $\Pi$ becomes a real matrix. In this case, the stability robustness condition can be carried as a linear matrix inequality by direct application of the KYP lemma [26].

\subsection{Additive uncertainty}

The corresponding condition in Theorem 1 against additive norm-bounded uncertainty can be obtained by setting $G_{11}=0$ and $G_{12}=G_{21}=\Gamma=I$. In addition, sector-bounded conditions to represent the dead zone nonlinearity are obtained by setting $W$ as diagonal, real and positive definite. In this case, the term $L_{S}$ becomes

$$
L_{S}=\left[\begin{array}{cc}
M^{*} M-M^{*} W-W M & \left(W-M^{*}\right) H \\
H^{*}(W-M) & H^{*} H-\gamma_{\Delta}^{2} I
\end{array}\right]
$$

Such plant uncertainty and dead zone descriptions have also been considered in [13]. In this work, the authors state that the Weston-Potlethwaite is guaranteed robustly stable if there exist some symmetric matrix $P>0$, diagonal $W>0$ and sufficiently small $\gamma_{J}>0$ such that

$$
\begin{array}{r}
\frac{d}{d t} x^{T} P x+\|z\|^{2}-\gamma_{J}^{2}\left\|u_{l i n}\right\|^{2}+2 \tilde{u}^{T} W\left(u_{l i n}-u_{d}-\tilde{u}\right)<0 \\
\gamma_{J}\|H\|_{\infty}<\gamma_{\Delta}
\end{array}
$$


where $x$ is the state vector associated with $M$. This condition can also be obtained (see Appendix section) by means of the IQC result (Lemma 1) with the following elements in the standard feedback interconnection

$$
\begin{aligned}
p & =\left[\begin{array}{c}
p_{d z} \\
z
\end{array}\right], q=\left[\begin{array}{c}
q_{d z} \\
u_{\text {lin }}
\end{array}\right] \\
\Phi & =\left[\begin{array}{ll}
\mathrm{dz} & \\
& (H \Delta)
\end{array}\right], F=\left[\begin{array}{cc}
I-M & I \\
-M & I
\end{array}\right]
\end{aligned}
$$

which, expressed in the frequency domain, becomes

$$
\left[\begin{array}{cc}
M^{*} M-W M-M^{*} W & \left(W-M^{*}\right) H \\
H^{*}(W-M) & H^{*} H\left(1-\gamma_{J}^{2}\right)
\end{array}\right]<0, \text { for all } \omega
$$

Because of the operator

$$
\left[\begin{array}{cc}
\mathrm{dz} & \\
& (H \Delta)
\end{array}\right]=\left[\begin{array}{ll}
I & \\
& H
\end{array}\right]\left[\begin{array}{ll}
\mathrm{dz} & \\
& \Delta
\end{array}\right]
$$

it follows immediately by a simple small gain argument that condition (22) implies the corresponding condition in Theorem 1; hence, it is more conservative. Despite the increased level of conservatism, the appeal of condition (22) lies in its simplicity, which is advantageous for antiwindup synthesis.

\section{PRESERVATION OF ROBUSTNESS}

This section forms the main part of the manuscript and is concerned with the preservation of robustness of the studied anti-windup schemes. An anti-windup system preserves robustness if the saturated loop is as robust as the unconstrained loop. To determine whether a system with saturations and anti-windup preserves the robustness of its unconstrained counterpart, it is sufficient to investigate if there exist valid choices for the anti-windup elements such that the condition for robustness of the linear system implies the robustness condition of the saturated loop.

The work [13] discusses the concept and observes that any such robustness-preserving antiwindup system must be optimally robust. For the case of unstructured additive uncertainty, [13] shows that the conventional IMC structure (where $M=I$ ) is optimally robust, in the sense that if the unsaturated loop is robust to uncertainty, satisfying

$$
\|\Delta p\|^{2} \leqslant\|p\|^{2} / \gamma_{\Delta}^{2}
$$

then setting $M=I$ ensures the loop with saturations is also robust to such uncertainty. See also [27].

We generalise the results on robustness-preserving anti-windup to structured norm-bounded uncertainty. In particular, we allow uncertainty of the form (1) where $\Delta$ satisfies (2). When there is no saturation, we have

$$
p_{\Delta}=\Theta q_{\Delta}
$$

with $\Theta$ given by (18). It follows that the unsaturated loop is robustly stable provided

$$
\Theta^{*} \Gamma \Theta-\gamma_{\Delta}^{2} \Gamma<0
$$

for all $\omega$. We may write this condition $L_{22}(j \omega)<0$ for all $\omega$ where $L_{22}$ is defined in (17). So our anti-windup scheme defined by $M$ preserves robustness if $L_{22}(j \omega)<0$ for all; $\omega$ implies there exists a valid choice for $W$ such that $L_{S}(j \omega)<0$ with $L_{S}(j \omega)$ defined by (16) and (17). 
Theorem 2

The Weston-Postlethwaite anti-windup structure is guaranteed optimally robust if

- There exists an admissible IQC multiplier $\Gamma>0$ such that

$$
G_{12}^{*} \Gamma G_{12}+\bar{G}_{11}^{*} \Gamma G_{12}+G_{12}^{*} \Gamma \bar{G}_{11}>0, \text { for all } \omega
$$

for some $\bar{G}_{11}$ such that

$$
G_{11}=\bar{G}_{11} H G_{21}
$$

- The multiplier

$$
W=M^{*} G_{12}^{*} \Gamma\left(G_{12}+\bar{G}_{11}\right)
$$

is admissible with $M$ being stable and invertible and satisfying $M(\infty)=I$.

Proof

This choice of $W$ yields

$$
\begin{aligned}
& L_{21}=0 \\
& L_{11}=-M^{*}\left(G_{12}^{*} \Gamma G_{12}+\bar{G}_{11}^{*} \Gamma G_{12}+G_{12}^{*} \Gamma \bar{G}_{11}\right) M
\end{aligned}
$$

We can then apply Theorem 1 .

By using such choices for $\bar{G}_{11}$ and $W$, the original robustness condition for the anti-windup system becomes equivalent to that when saturations are non existent, that is, condition (16) becomes equivalent to (27). Therefore, it can be claimed that robustness preservation of the unsaturated loop is achieved.

In most applications, the uncertainty description of the plant is prescribed together with the linear controller. In this case, $\bar{G}_{11}$ is uniquely determined from (29) because $G_{11}, G_{21}$ and $H$ are all given. $\bar{G}_{11}$ will not exist if $\operatorname{det}\left(H G_{21}(j \omega)\right)=0 \forall \omega$, and hence, robustness preservation can not be guaranteed. More generally, the condition for the existence of $\bar{G}_{11}$ is that the rows of $G_{11}$ are in the space spanned by the rows of $H G_{21}$. When $H G_{11}$ is invertible, this condition is satisfied automatically.

If $\bar{G}_{11}$ exists so (29) holds, and $M$ is obtained following certain nominal anti-windup design rules, optimal robustness guarantees will depend then on choosing an admissible $\Gamma$ so (30) and (28) are valid. In some cases, the search of an admissible set of $\Gamma$ can be straightforward, as shown in Example 1 later in the text. In other cases, however, the search over multipliers $\Gamma$ and $W$ is not. This is the usual trade-off between conservatism and simplicity one encounters when performing IQC-based robustness tests with dynamic multipliers.

\section{Corollary}

If $H G_{21}$ is invertible, then the architecture in Figure 3 retains the robustness of the unconstrained loop if for some $\Gamma>0$,

$$
\Theta^{*} \Gamma \Theta-G_{11}^{*} \Gamma G_{11}>0 \text { for all } \omega
$$

and the multiplier

$$
W=M^{*} G_{12}^{*} \Gamma\left(G_{12}+G_{11}\left(H G_{21}\right)^{-1}\right)
$$

is admissible with $M$ being stable and invertible and satisfying $M(\infty)=I$.

Proof

Substitute $\bar{G}_{11}=G_{11}\left(H G_{21}\right)^{-1}$ and premultiply and post-multiply inequality (28) by $\left(H G_{21}\right)^{*}$ and $H G_{21}$, respectively. 
The condition for optimal robustness in the Corollary is much simpler because there is no need for the term $\bar{G}_{11}$. Note also that there might not exist an anti-windup choice of $M$ for which robustness preservation is guaranteed given a prescribed uncertainty description and a linear control design. If such an anti-windup design is not possible to be found via Theorem 2, then there might exist a choice of $M(s)$ for which robustness might be ensured but for a smaller uncertainty size. In this sense, a 'smaller uncertainty size' is represented by a larger value of $\gamma_{\Delta}$ or by a corresponding scaling of the uncertainty descriptors $G_{11}$ and $G_{12}$. Theorem 2 can be used to determine numerically the reduction in size of the uncertainty given a fixed anti-windup design by iterating over $\gamma_{\Delta}$.

\section{EXAMPLES AND COUNTEREXAMPLES}

To corroborate the ideas exposed so far, we provide later in the text three examples and one counterexample. Examples 1-3 show cases of robustness-preserving anti-windup schemes, which are not necessarily the conventional IMC loop for the case of additive uncertainty. Counterexample 1 illustrates the case where the conventional IMC structure does not preserve robustness against output multiplicative uncertainty.

\section{Example 1}

This example illustrates the application of Theorem 2 to investigate the robustness-preserving properties of the following choice of anti-windup

$$
Q_{f}=\lambda Q+(1-\lambda) Q(\infty)
$$

against additive uncertainty $\left(G_{11}=0, G_{12}=G_{21}=1\right)$ for the SISO case. The parameter $\lambda \in[0,1]$ is used to trade-off between robustness and performance, refer to [15] for details. Notice that the choice $\lambda=1$ corresponds to the conventional IMC structure.

Assume the plant model is given by first-order dynamics with a delay

$$
G_{22}(s)=e^{-s d} \frac{b}{s+a}
$$

with $a, b, d>0$. Application of linear IMC design methodologies [28] leads to

$$
Q(s)=\frac{c}{b} \frac{s+a}{s+c}
$$

with $c>0$. The design rule (34) produces the following anti-windup element

$$
M(s)=\frac{s+a}{s+\bar{c}}
$$

where $\bar{c}=\lambda a+(1-\lambda) c>0$. Notice that the above $M$ is invertible and $M(\infty)=1$.

Given the previous scenario, application of Theorem 2 guarantees that the robustness of the linear loop, described by

$$
\|H\|_{\infty}=\|Q\|_{\infty}=\max \{a / b, c / b\}<\gamma_{\Delta}
$$

is preserved if for any constant $\Gamma>0$, the multiplier

$$
W(s)=\Gamma M(s)=\Gamma\left(1+\frac{a-\bar{c}}{s+\bar{c}}\right)
$$

is valid. In terms of the Zames-Falb requirements, the condition becomes

$$
\Gamma \int_{-\infty}^{\infty}|\delta(t)+f(t)| d t<1
$$

with $\delta(t)$ being the Dirac delta function and

$$
f(t)= \begin{cases}(a-\bar{c}) e^{-\bar{c} t} & , t \geqslant 0 \\ 0 & , t<0\end{cases}
$$


Simplification of the previous condition translates that by choosing any $\Gamma$ such that

$$
0<\Gamma<\frac{\bar{c}}{a}
$$

and multiplier (39), the conditions in Theorem 2 are satisfied, that is, robustness is guaranteed to be preserved for the class of anti-windup controllers (34) where $\lambda \in[0,1]$.

\section{Example 2}

Assume $G_{11}=0$ (additive or multiplicative plant uncertainty) and $G_{12}^{*} \Gamma G_{12}>0$ for all $\omega$. Suppose we take the spectral factorisation

$$
G_{12}^{*} \Gamma G_{12}=\Phi^{*} \Phi
$$

where $\Phi$ is causal, stable and minimum phase and set

$$
W=\Phi^{*}(\infty) \Phi
$$

If $W$ is an admissible multiplier, then we can choose

$$
M=\Phi^{-1} \Phi(\infty)
$$

In particular, we find $M(\infty)=I, M$ is both stable and minimum phase, and with this choice $L_{11}=-\Phi^{*}(\infty) \Phi(\infty)$ and $L_{21}=0$. So $L_{S}<0$ for all $\omega$, that is, the anti-windup loop preserves robustness.

\section{Example 3}

If $G_{11}=0, G_{12}$ is diagonal with scalar elements and $\Gamma$ is diagonal, then the construction of Example 2 yields $W$ with scalar diagonal elements. Hence, $W$ is admissible. Furthermore, $M=I$, so the conventional IMC scheme is shown to preserve robustness, is also against diagonal uncertainty.

The authors in [13] state that their results can be generalised in a straightforward manner to output multiplicative uncertainty (where $G_{11}=0, G_{21}=I, G_{12}=G_{22}$ and $\Gamma=I$ ) and input multiplicative uncertainty (where $G_{11}=0, G_{12}=I, G_{21}=G_{22}$ and $\Gamma=I$ ). Example 3 confirms their statement for input multiplicative uncertainty. However, their statement for output multiplicative uncertainty is not true: the conventional IMC arrangement is not guaranteed to preserve robustness in this case (we have no guarantee that $L_{S}<0$ for all $\omega$ ). See Figure 6 where NL: $u_{l i n} \mapsto z$.

\section{Counterexample 1}

This counterexample is constructed under the IMC anti-windup scheme. Assume a two-input two-output plant with nominal model

$$
G_{22}(s)=g(s)\left[\begin{array}{cc}
1 & x(s) \\
0 & 1
\end{array}\right]
$$

where both $g(s)$ and $x(s)$ are SISO transfer functions. Similarly, we will choose $Q$ to have structure

$$
Q(s)=q(s)\left[\begin{array}{cc}
1 & -x(s) \\
0 & 1
\end{array}\right]
$$

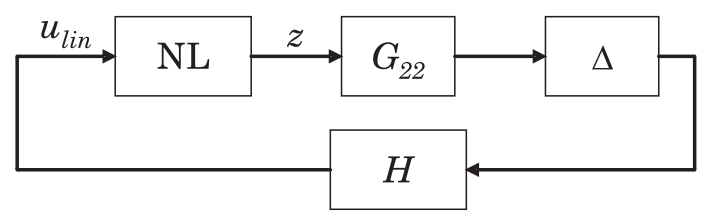

Figure 6. Block diagram of the Weston-Postlethwaite structure with output multiplicative uncertainty. 


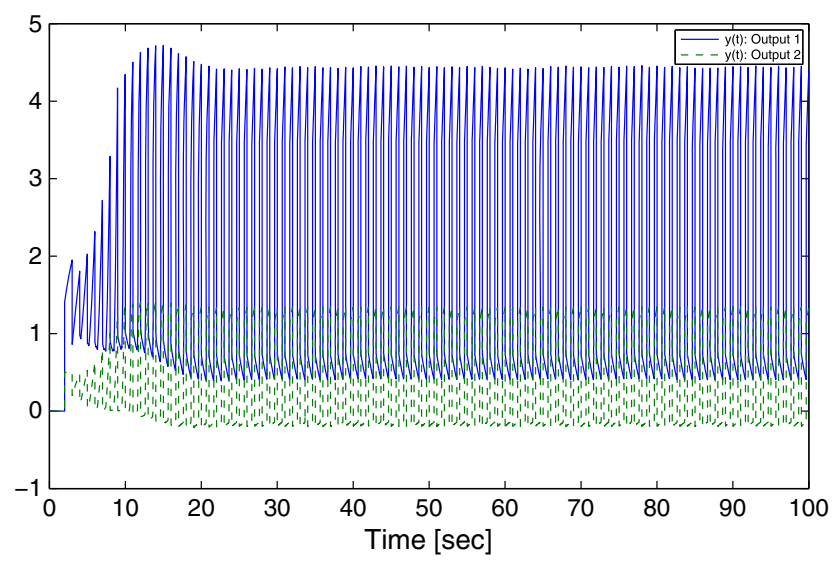

Figure 7. Limit cycling behaviour for internal model control anti-windup with output multiplicative uncertainty.

where $q(s)$ is also a SISO transfer function. Then

$$
Q(s) G(s)=q(s) g(s) I
$$

We will choose $q(s)$ such that $q(0) g(0)=1$ and $\|q g\|_{\infty}=1$. For output multiplicative uncertainty, we choose $G_{21}=I$ and $G_{12}=G_{22}$. It follows that the unsaturated loop is robust to output multiplicative uncertainty provided

$$
\gamma_{\Delta}>1
$$

Set

$$
\begin{aligned}
& x(s)=5.6 \frac{s+1}{2 s+1} \\
& g(s)=e^{-s} \\
& q(s)=1
\end{aligned}
$$

and

$$
\begin{aligned}
Q_{f}(s) & =Q(s) \\
Q_{b}(s) & =0
\end{aligned}
$$

In addition, choose

$$
\Delta=\frac{1}{2}\left[\begin{array}{ll}
0 & 1 \\
1 & 0
\end{array}\right]
$$

The output signals from a simulation of this system are shown in Figure 7. The saturation levels were set at \pm 1 for each manipulated variable. A unit step-up was applied to the set point for the first output at time $t=1$ and a unit step down at time $t=2$. It is clear that there is a limit cycle.

\section{CONCLUSIONS}

The main outcomes of the paper are as summarised:

1. Robustness-preserving characteristics of two popular anti-windup schemes have been discussed in this paper under the IQC framework. Existing results have been generalised to structured norm-bounded uncertainty, which are more useful for practical purposes. 
2. It is shown that the conventional IMC structure need not preserve robustness except for very specific uncertainty structures.

3. We have used 1. to derive examples where anti-windup structures other than IMC preserve robustness.

The condition for robustness preservation is given in terms of IQC multipliers for descriptions of the uncertainty and the nonlinear operator. In some cases, such multipliers can be easily chosen as shown in this paper. However, this is not the case for the multivariable case. Anti-windup design involves a trade-off between (robust) stability and performance [13,29], and choice of structure is likely to be as important as analytical method. Current research explores ways to exploit these results for the aim of anti-windup design with robustness-preserving properties and systematic selection of multipliers.

\section{APPENDIX. ALTERNATIVE DERIVATION OF THE ROBUSTNESS CONDITION OF [13] FOR ADDITIVE UNCERTAINTY.}

Turner et al. [13] obtain the sufficient robustness condition in (22) for the Weston-Postlethwaite anti-windup structure for the case of additive uncertainty. Such a condition can also be expressed as an inequality in the frequency domain and the equivalence can be established via the KYP lemma [30]. For this purpose, let $M$ have the following state space representation

$$
M \sim\left[\begin{array}{l|l}
A & B \\
C & I
\end{array}\right]
$$

then noting that

$$
\begin{aligned}
\frac{d}{d t} x & =A x+B \tilde{u} \\
z & =u_{l i n}-C x-\tilde{u} \\
u_{d} & =C x
\end{aligned}
$$

we obtain the equivalent LMI condition that

$$
\left[\begin{array}{ccc}
P A+A^{T} P+C^{T} C & P B+C^{T}-C^{T} W & -C^{T} \\
B^{T} P+C-W C & I-2 W & W-I \\
-C & W-I & I\left(1-\gamma_{J}^{2}\right)
\end{array}\right]<0
$$

By the KYP lemma, this is equivalent to the frequency domain inequality

$$
\begin{aligned}
{\left[\begin{array}{cc}
(j \omega I-A)^{-1} B & 0 \\
I & 0 \\
0 & I
\end{array}\right]^{*} } & {\left[\begin{array}{ccc}
C^{T} C & C^{T}-C^{T} W & -C^{T} \\
C-W C & I-2 W & W-I \\
-C & W-I & I\left(1-\gamma_{J}^{2}\right)
\end{array}\right] } \\
& \times\left[\begin{array}{ccc}
(j \omega I-A)^{-1} B & 0 \\
I & 0 \\
0 & I
\end{array}\right]<0 \text { for all } \omega
\end{aligned}
$$

The previous expression can also be expressed as

$$
\left[\begin{array}{cc}
M^{*} M-W M-M^{*} W & W-M^{*} \\
W-M & I\left(1-\gamma_{J}^{2}\right)
\end{array}\right]<0 \text { for all } \omega
$$

Premultiplying by $\operatorname{diag}\left(I, H^{*}\right)$ and post-multiplying by $\operatorname{diag}(I, H)$ reduce the stability condition to condition (24). In terms of Lemma 1, this is the robustness condition that is obtained when the elements in the feedback loop are those expressed in (23). The condition derived in this work (21) is less conservative. 


\section{REFERENCES}

1. Morales RM, Li G, Heath WP. Anti-windup and the preservation of robustness against structured norm-bounded uncertainty. IFAC World Congress, Seoul, Korea, 2008; 14144-14149.

2. Heath W, Morales R, Li G. Robustness preserving antiwindup: examples and counterexamples. Proceedings of the IEEE Multiconference on Systems and Control, Denver, USA, 2011; 204-209.

3. Aström KJ, Wittenmark B. Computer Controlled Systems: Theory and Design. Prentice Hall: New Jersey, USA, 1984.

4. Hanus R, Kinnaert M, Henrotte J-L. Conditioning technique, a general anti-windup and bumpless transfer method. Automatica 1987; 23(6):729-739.

5. Campo PJ, Morari M. Robust control of processes subject to saturation nonlinearities. Computers \& Chemical Engineering 1990; 14(4/5):343-358.

6. Mulder EF, Kothare MV, Morari M. Multivariable anti-windup controller synthesis using linear matrix inequalities. Automatica 2001; 37:1407-1416.

7. Teel AR, Kapoor N. The $\mathcal{L}_{2}$ anti-windup problem: its definition and solution. Proceedings of the Fourth European Control Conference, Brussels, Belgium, 1997; 120-128.

8. Zaccarian L, Teel AR. A common framework for anti-windup, bumpless and reliable designs. Automatica 2002; 38: $1735-1744$.

9. Grimm G, Hatfield J, Postlethwaite I, Teel AR, Turner MC, Zaccarian L. Anti-windup for stable linear systems with input saturations: An LMI-based approach. IEEE Transactions on Automatic Control 2003; 48(9):1509-1525.

10. Kothare MV, Morari M. Multiplier theory for stability analysis of anti-windup control systems. Automatica 1999; 35:917-928.

11. Megretski A, Rantzer A. System analysis via integral quadratic constraints. IEEE Transactions on Automatic Control 1997; 42(6):819-830.

12. Jönsson U, Rantzer A. Optimization of integral quadratic constraints. In Advances in Linear Matrix Inequality Methods in Control, El Ghaoui L, Niculescu S-I (eds). SIAM: Philadelphia, 2000; 109-127.

13. Turner MC, Herrmann G, Postlethwaite I. Incorporating robustness requirements into antiwindup design. IEEE Transactions on Automatic Control 2007; 52(10):1842-1855.

14. Weston PF, Postlethwaite I. Linear conditioning for systems containing saturating actuators. Automatica 2000; 36:1347 -1354.

15. Zheng A, Kothare MV, Morari M. Anti-windup design for internal model control. International Journal of Control 1994; 60(5):1015-1024.

16. McFarlane D, Glover K. Robust Controller Design Using Normalised Coprime Factor Plant Descriptions. Springer Verlag: Berlin, 1990.

17. Vinnicombe G. Uncertainty and Feedback. Imperial College Press: London, 2001.

18. Skogestad S, Postlethwaite I. Multivariable Feedback Control: Analysis and Design, (second edition). John Wiley \& Sons: Chichester, England, 2005.

19. Jönsson U. Lecture notes on integral quadratic constraints, 2001. Department of Mathematics, KTH, Stockholm. ISBN 1401-2294, (Available from: http://www.math.kth.se/ uj/) [accessed on July 2011].

20. Khalil HK. Nonlinear Systems, (third edition). Prentice Hall: Upper Saddle River, 2002.

21. Morari M, Zafirou E. Robust Process Control. Prentice Hall: Englewood Cliffs, 1989.

22. Kothare MV, Campo PJ, Morari M, Nett CN. A unified framework for the study of anti-windup designs. Automatica 1994; 30(12):1869-1883.

23. Zames G, Falb PL. Stability conditions for systems with monotone and slope-restricted nonlinearities. SIAM Journal of Control 1968; 6(1):89-108.

24. D'Amato FJ, Rotea MA, Megretski AV, Jönsson UT. New results for analysis of systems with repeated nonlinearities. Automatica 2001; 37(5):739-747.

25. Mancera R, Safonov MG. All stability multipliers for repeated MIMO nonlinearities. Systems and Control Letters 2005; 54(4):389-397.

26. Rantzer A. On the Kalman-Yakubovich-Popov lemma. Systems and Control Letters 1996; 28:7-10.

27. Morales RM. The robustness of constrained cross-directional control and anti-windup systems. Ph.D. Thesis, Control Systems Research Group, School of Electrical and Electronic Engineering, The University of Manchester, Manchester, UK, 2008.

28. Garcia CE, Morari M. Internal model control 1. A unifying review and some new results. Industrial \& Engineering Chemistry Process Design and Development 1982; 21(2):308-323.

29. Galeani S, Teel AR. On performance and robustness issues in the anti-windup problem. Proceedings of the 43rd Conference on Decision and Control, Paradise Island, Bahamas, 2004; 5022-5027.

30. Boyd S, Ghaoui LE, Feron E, Balakrishnan V. Linear Matrix Inequalities in Systems and Control Theory, Studies in Applied Mathematics. SIAM: Philadelphia, PA, 1994. 